\title{
Two-Photon Absorption in microcavties for optical autocorrelation and sampling
}

\author{
T.Krug, M.Lynch, H. Folliot, A.L. Bradley and J.F.Donegan \\ Semiconductor Photonics Group. Physics Department. Trinity College, Dublin 2, Ireland \\ . T:00.353-1-6082/67, F:00353-1.6082680,E: krugf@ttd.ie \\ L.P. Barry \\ Department of Electronic Engineering, Dublin City University, Dublin 9, Ireland \\ Liam.barry@dcu.ie \\ J.S. Roberts and G. Hill \\ Department of Electronic and Electrical Engineering, University of Sheffield, Mappin Street, Sheffield, SI 3JD, England
}

We have designed novel semiconductor microcavity structures for the enhancement of the two-photon absorption (TPA) photocurrent. This proof of concept device is a monolithic semiconductor microcavity with a $0.27 \mu \mathrm{m} \mathrm{Ga}_{0.7} \mathrm{Al}_{0.3 .3} \mathrm{As}$ active region and two Bragg reflectors with the cavity resonance of $890 \mathrm{~nm}$. The effect of the cavity is to enhance the intra-cavity optical intensity, which leads to an increase in the non-linear response of the active region. We have measured a factor of 12000 enhancement in TPA photocurrent over a non-microcavity device at $890 \mathrm{~nm}$.

We report a TPA autocorrelation technique for short optical pulses that uses the microcavity structure instead of a second harmonic generation crystal. The laser pulse is launched into a standard Michelsontype autocorrelator and focused by a lens onto the TPA microcavity device. Comparison of the measured pulsewidth with a $\mathrm{BBO}$ crystal response shows only small broadening of the pulse due to the photon cavity lifetime.

We also measured the depth-of-field response and varied the incident angle of the laser pulse focusing onto the device. Because of microcavity effects the behaviour was observed to be different to second harmonic generation. Knowledge of these characteristics is important for implementation in applications such as optical switching and sampling in optical time division multiplexed (OTDM) communications systems.

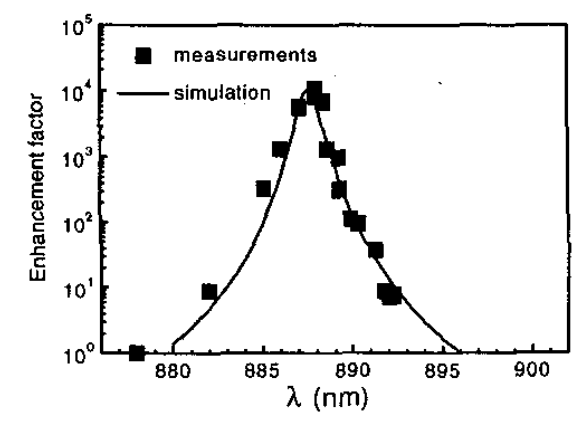

Fig.1. Photocurrent enhancement vs. wavelength across the cavity resonance. 